\title{
Efficacy of ALK5 inhibition in myelofibrosis
}

\author{
Lanzhu Yue,${ }^{1,2}$ Matthias Bartenstein, ${ }^{3}$ Wanke Zhao, ${ }^{4}$ Wanting Tina Ho, ${ }^{4}$ Ying Han, ${ }^{1,5}$ \\ Cem Murdun, ${ }^{1}$ Adam W. Mailloux, ${ }^{6}$ Ling Zhang, ${ }^{7}$ Xuefeng Wang, ${ }^{8}$ Anjali Budhathoki, ${ }^{3}$ \\ Kith Pradhan, ${ }^{3}$ Franck Rapaport, ${ }^{9}$ Huaquan Wang, ${ }^{2}$ Zonghong Shao, ${ }^{2}$ Xiubao Ren, ${ }^{5}$ Ulrich Steidl, ${ }^{3}$ \\ Ross L. Levine, ${ }^{9}$ Zhizhuang Joe Zhao, ${ }^{4}$ Amit Verma, ${ }^{3}$ and Pearlie K. Epling-Burnette ${ }^{1}$ \\ 'Department of Immunology, H. Lee Moffitt Cancer Center and Research Institute, Tampa, Florida, USA. ${ }^{2}$ Department \\ of Hematology, Tianjin Medical University General Hospital, Tianjin, China. ${ }^{3}$ Department of Oncology, Albert Einstein \\ College of Medicine, Montefiore Medical Center, Bronx, New York, USA. ${ }^{4}$ Department of Pathology, Peggy and Stephenson \\ Cancer Center, Oklahoma University Health Sciences Center, Oklahoma City, Oklahoma, USA. ${ }^{5}$ Department of Biotherapy, \\ Tianjin Medical University Cancer Institute and Hospital, National Clinical Research Center of Cancer, Key Laboratory of \\ Cancer Immunology and Biotherapy, Key Laboratory of Cancer Prevention and Therapy, Tianjin, PR China. ${ }^{6}$ Translational \\ Science, H. Lee Moffitt Cancer Center and Research Institute, Tampa, Florida, USA. ${ }^{7}$ Department of Hematopathology and \\ Laboratory Medicine, ${ }^{8}$ Department of Biostatistics and Bioinformatics, H. Lee Moffitt Cancer Center \& Research Institute, \\ Tampa, Florida, USA. ' ${ }^{2}$ eukemia Center, Memorial Sloan Kettering Cancer Center, New York City, New York, USA.
}

Myelofibrosis (MF) is a bone marrow disorder characterized by clonal myeloproliferation, aberrant cytokine production, extramedullary hematopoiesis, and bone marrow fibrosis. Although somatic mutations in JAK2, MPL, and CALR have been identified in the pathogenesis of these diseases, inhibitors of the Jak2 pathway have not demonstrated efficacy in ameliorating MF in patients. TCF- $\beta$ family members are profibrotic cytokines and we observed significant TCF- $\beta 1$ isoform overexpression in a large cohort of primary MF patient samples. Significant overexpression of TCF- $\beta 1$ was also observed in murine clonal MPL ${ }^{\text {W515L }}$ megakaryocytic cells. TGF- $\beta 1$ stimulated the deposition of excessive collagen by mesenchymal stromal cells (MSCs) by activating the TCF- $\beta$ receptor I kinase (ALK5)/Smad3 pathway. MSCs derived from MPL ${ }^{W 515 L}$ mice demonstrated sustained overproduction of both collagen I and collagen III, effects that were abrogated by ALK5 inhibition in vitro and in vivo. Importantly, use of galunisertib, a clinically active ALK 5 inhibitor, significantly improved MF in both $M P L^{\text {W515L }}$ and JAK2 ${ }^{\mathrm{V} 617 F}$ mouse models. These data demonstrate the role of malignant hematopoietic stem cell (HSC)/TCF- $\beta$ /MSC axis in the pathogenesis of MF, and provide a preclinical rationale for ALK5 blockade as a therapeutic strategy in MF.

Authorship note: L. Yue, M. Bartenstein, and W. Zhao contributed equally to this work.

Conflict of interest: The authors have declared that no conflict of interest exists.

Submitted: October 4, 2016 Accepted: February 1, 2017 Published: March 23, 2017

Reference information: JCl Insight. 2017;2(6):e90932. https:// doi.org/10.1172/jici.insight.90932.

\section{Introduction}

Philadelphia-negative myeloproliferative neoplasms (MPNs) comprise multipotent stem cell disorders including polycythemia vera (PV), essential thrombocythemia (ET), and primary myelofibrosis (PMF) (1). These disorders are characterized by clonal expansion of myeloid lineage cells due to constitutive activation of the JAK/STAT pathway driven by several somatic mutations in JAK2, MPL, and CALR genes (2-4). Myelofibrosis $(\mathrm{MF})$, either presenting as PMF or evolving from PV or ET secondarily, is exemplified by remodeling of bone marrow stroma towards excessive deposition of extracellular matrix (ECM), neoangiogenesis, and osteosclerosis. These changes collectively result in substitution of normal bone marrow niche proteins and factors with dense fibrillar matrix $(5,6)$. MF patients suffer from a series of symptoms such as splenomegaly, hepatomegaly, severe anemia, bleeding, and thrombosis caused by bone marrow fibrosis and extramedullary hematopoiesis, which decrease both quality of life and survival. Currently there are limited treatment options for MF, although allogeneic stem cell transplantation provides remissions in younger patients $(7,8)$. Curative therapies may be lacking, owing, in part, to the poorly understood bone marrow niche-related pathogenesis of MF.

It has been hypothesized that the development of $\mathrm{MF}$ is attributable to aberrant interactions between neoplastic hematopoietic clones and bone marrow niche-comprising mesenchymal stromal cells (MSCs) and their differentiated counterparts, adipocytes, chondrocytes, and osteoblasts. Neoplastic clones might 
modify the bone marrow niche by releasing fibrotic and angiogenic cytokines such as platelet factor 4 (PF4), TGF- $\beta 1$, PDGF, and VEGF (6, 9-13). However, it is still not fully understood what the pathologic changes are in bone marrow stromal cells and how they participate in MF pathogenesis and survival. In our previous studies, MSC cultures have been informative in understanding the mechanisms leading to excessive collagen production in patients' bone marrow (14), which may be useful to evaluate therapeutics that target these pathways specifically.

Among the abnormally expressed cytokines in MF, TGF- $\beta 1$ has received attention due to its critical role in inducing fibrosis not only in bone marrow (15-18), but other organs (19-21). TGF- $\beta 1$ may be produced by abnormal megakaryocytes or monocytes, and this in turn may remodel the MSCs that promote the changes illustrated by MF (22-25). In this study, galunisertib (LY2157299) (26), a TGF- $\beta$ receptor I kinase (ALK5) inhibitor, was used to show that TGF- $\beta 1$ indeed alters the bone marrow microenvironment through modulation of MSCs. Since galunisertib is an ALK5 inhibitor, it is possible that TGF- $\beta 1$ or other TGF- $\beta$-family proteins induce the fibrotic phenotype in MF mouse models. Collectively, blockade of TGF- $\beta$ signaling by galunisertib reverses the fibrotic MSC phenotype and restores the microenvironment in animal models of MF.

\section{Results}

$T G F-\beta 1$ is overexpressed in human PMF samples and $M P L^{W 515 L}$ mice. Expression patterns of various TGF ligands in human MPN neutrophil samples were first examined in a large cohort of samples representing PV, ET, and MF (27). TGFB1 mRNA was significantly overexpressed compared with TGF 2 and TGF 3 mRNA when compared with healthy controls $(P=0.03)$ (Figure 1A). A subgroup analysis in the MPN cohort demonstrated that TGFB1 overexpression relative to control was highest in MF samples $(n=18, P=0.002)$, followed by samples from patients with PV $(n=28)$ and ET $(n=47)$ (Figure 1B). Expression of the Tgfb1 isoform was also markedly increased in bone marrow mononuclear cells from mice on day 17 after transplantation with $M P L^{W 515 L}$ bone marrow compared with those from $M P L^{W T}$ recipients $(P<0.0001)$ (Figure 1C). Furthermore, mouse bone marrow hematopoietic cells transduced in vitro with $M P L^{W 515 L}$ retrovirus displayed significantly higher $T g f b 1$ mRNA expression compared with those expressing $M P L^{W T}$ virus $(P=$ 0.0024) (Figure 1D).

Since megakaryocytes are known to be major source of TGF- $\beta$ secretion (24), we analyzed whether numbers of megakaryocytes were increased in the mice transplanted with $M P L^{W 515 L}$-transduced cells that coexpressed GFP. Megakaryocytes were determined by anti-CD41 antibody staining and platelets were stained with anti-CD42 (glycoprotein $\mathrm{Ib}, \mathrm{GP} 1 \mathrm{~b}$ ). We observed a significantly higher number of CD41 ${ }^{+}$ megakaryocytes in the $M P L^{W 515 L}$ mice when compared with controls (Figure 1, E and F). The CD41 ${ }^{+}$cells expressed GFP, indicating derivation from the mutant $M P L^{W 515 L}$ stem and progenitor population (Figure 1, $\mathrm{G}-\mathrm{I})$. Sorting and expression analysis of these megakaryocytes demonstrated that $\mathrm{GFP}^{+} \mathrm{CD} 41^{+}$cells that arose from the mutant clone displayed a significantly higher expression of $T g f b 1 \mathrm{mRNA}$ when compared with WT GFP- megakaryocytes (Figure 1J). These data confirm that TGF 1 is increased in human MF as well as in a mouse model based on expression of the mutant $M P L^{W 515 L}$ oncogene. The increased expression in $T g f b 1$ in the mouse model is collectively due to higher numbers of megakaryocytes as well as greater secretion of $T g f b 1$ by mutant megakaryocytes. This then prompted us to assess the therapeutic effect of TGF- $\beta$ blockade in mouse models of MF.

Galunisertib antagonizes TGF- $\beta 1$-stimulated collagen production by normal MSCs. Next, studies were performed on MSCs that were cultured from bone marrow of mice transplanted with $M P L^{W T}$ - and $M P L^{W 515 L}$ transduced grafts. MSCs derived from $M P L^{W T}$ mice and normal human bone marrow were cultured with recombinant murine or human TGF- $\beta 1$ together with variable doses of the clinical ALK5 inhibitor, galunisertib (28-30), for 72 hours and collagen (Col1A1 and Col3A1) mRNA expression was determined. Collagen I and III are clinically relevant since they are recognized by trichrome and reticulin staining, respectively. Our results show that TGF- $\beta 1$ led to induction of both Col1A1 and Col3A1 production in MSCs that was suppressed by the ALK5 inhibitor galunisertib in a dose-dependent manner (Figure 2, A and B). A similar result was seen using MSCs from normal human bone marrow (Figure 2, C and D). To confirm ECM deposition from cultured MSCs, immunofluorescence staining was also performed using multispectral fluorescence. Specificity of this staining with collagenase pretreatment was shown previously (14). We determined that excessive collagen deposition after TGF- $\beta 1$ treatment was reduced by ALK 5 inhibition (Figure 2, E and F). TGF- $\beta 1$ also led to activation of p-Smad3 in MSCs that was abrogated by galu- 


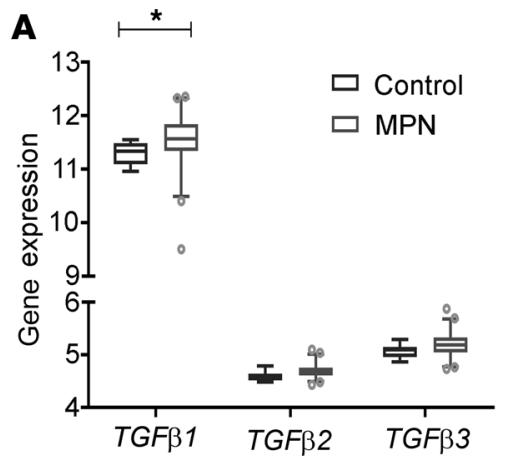

E

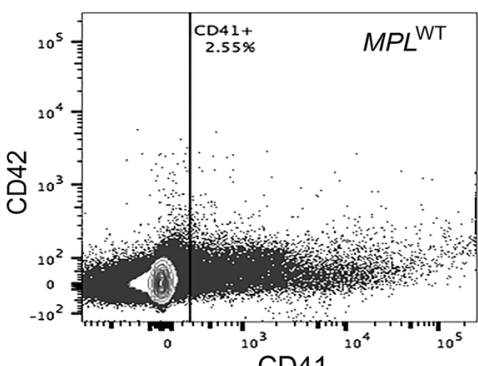

G

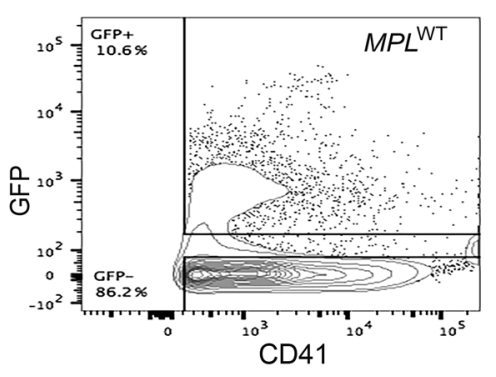

B

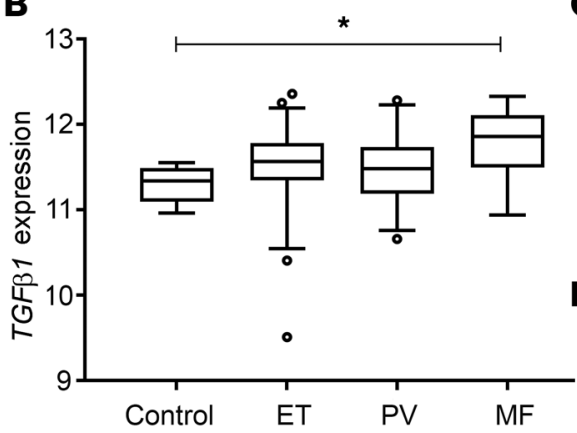

F

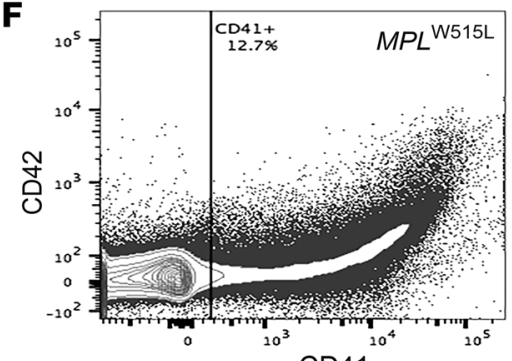

CD41

H

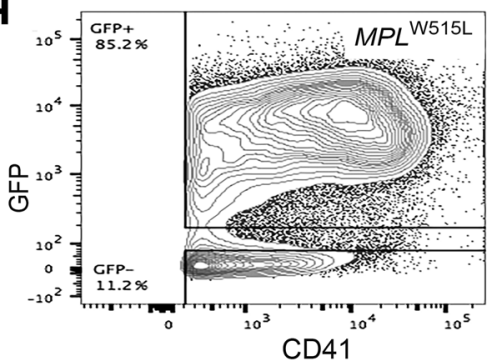

J

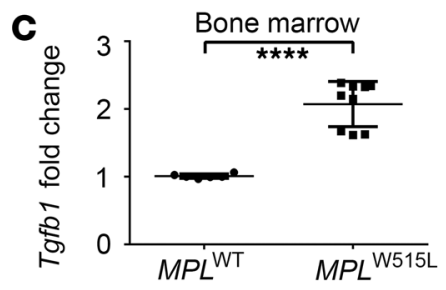

D

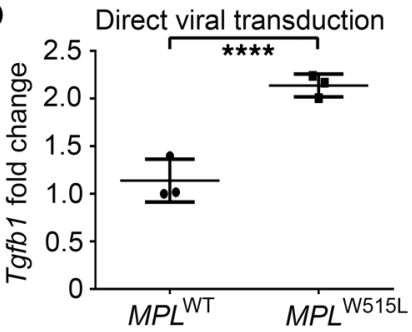

I
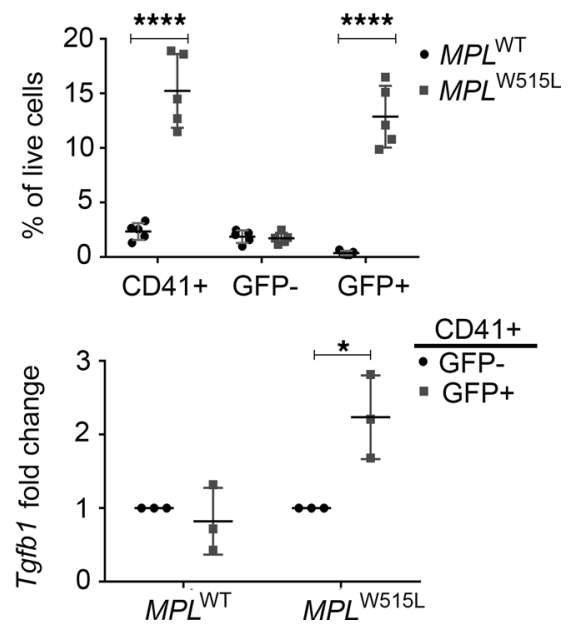

Figure 1. TGF- $\beta 1$ is overexpressed in human primary myelofibrosis samples and MPL $^{\text {W515L }}$ mice. (A and $\left.\mathbf{B}\right)$ Neutrophils derived from myeloproliferative neoplasm (MPN) patients were evaluated for expression of TCF $\beta$ family members by gene expression analysis (using Gapdh as reference gene). ${ }^{*} P<0.05$ by unpaired Student's $t$ test. (A) Analysis of TCF $\beta 1, T C F \beta 2$, and TCF $\beta 3$ mRNA expression in an MPN cohort compared with healthy donors (Control). ${ }^{*} P$ $<0.05$ as compared with control group by unpaired Student's $t$ test. (B) TCF $\beta 1$ mRNA expression was determined in MPN patients stratified by disease subtype including essential thrombocytosis (ET, $n=47$ ), polycythemia vera (PV, $n=28$ ), and myelofibrosis (MF, $n=18$ ). Data represent values relative to healthy control cells (Control). (C) qRT-PCR was performed for Tgfb1 mRNA using bone marrow mononuclear cells from MPL ${ }^{W T}$ and $M P L^{W 515 L}$ mice ( $n$ = 6). ${ }^{* * *} P<0.0001$ by unpaired 2-sided Student's $t$ test with Welch's correction. (D) Normal mouse bone marrow cells were directly transduced with retroviruses overexpressing either $M P L^{W T}$ or $M P L^{W 515 L}$, following which cells were collected and Tgfb1 mRNA was measured by $q R T-P C R$. ${ }^{* * * *} P<0.0001$ by unpaired 2-sided Student's $t$ test. (E-H) Representative contour flow cytometry plots of cells stained with antibodies against CD41 (clone HIP8) and CD42 (clone 1C2). GFP was simultaneously determined by flow cytometry and represents expression of GFP+ retroviral constructs ( $y$ axis, $\mathbf{G}$ and $\mathbf{H})$. The total CD41+ cells (E and $\mathbf{F}$ ) and GFP+CD41+ cells ( $\mathbf{G}$ and $\mathbf{H})$ in MPL ${ }^{\text {W515L }}$ spleens compared to MPL ${ }^{W T}$ controls. (I) Graph represents percentage of each population $\left(\mathrm{CD} 1^{+}, \mathrm{GFP}^{-}\right.$, and $\left.\mathrm{GFP}^{+}\right)$among live cells in the sample. Graphs (I and $\left.\mathbf{J}\right)$ represent cells from spleens of 5 individual mice with the mean of the population indicated as a line $\left(n=5 \mathrm{MPL}{ }^{\mathrm{WT}}\right.$ and $\left.n=5 \mathrm{MPL}{ }^{\mathrm{W} 515 \mathrm{~L}}\right)$. Statistical significance determined using the Holm-Sidak method, with $\alpha=5.000 \%$. ${ }^{* * *} P<0.0001$. (J) Fold change in normalized Tgf $\beta 1$ mRNA expression of CD41+CFP+ cells vs. Tgf $\beta 1$ expression in CD41+CFP- population from the same mouse ( $n=3$ MPL ${ }^{W T}$ and $\left.n=3 M P L^{W 515 L}\right) .{ }^{*} P<0.05$ by unpaired 2 -sided Student's $t$ test. All error bars indicate the mean \pm SD.

nisertib (Figure 2G). Since STAT3 has also been indicated in tissue fibrosis and potentially interacts with the TGF- $\beta$ pathway (31-33), STAT3 was also tested. TGF- $\beta 1$ failed to induce p-STAT3, but galunisertib slightly decreased this activation signal which could result from the modulation of a signaling network. These results indicate that galunisertib selectively suppresses TGF- $\beta$ signaling that is associated with the synthesis and deposition of collagen ECM in vitro in MSCs.

Elevated collagen production by MSCs derived from MPL ${ }^{W 515 L}$ mice is decreased by galunisertib in vitro. MPL ${ }^{W 515 L}$ mice developed enlarged spleens (Figure 3A) and severe MF (Figure 3B) within 17 days after transplantation with $M P L^{W S 15 L}$-transduced bone marrow. Increased collagen production was detected in MSCs derived from $M P L^{W 515 L}$ mice compared with those from $M P L^{W T}$ mice, as determined by quantitative reverse transcription PCR (qRT-PCR) (Figure 3, C and D) and immunofluorescence (Figure 3E). Since TGF- $\beta$ blockade sup- 


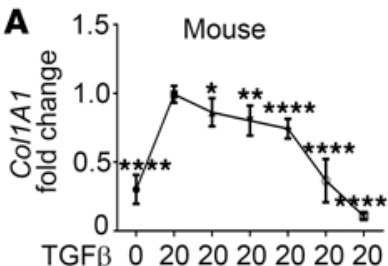

Galu $0 \quad 0.25 .5 \quad 1 \quad 510$
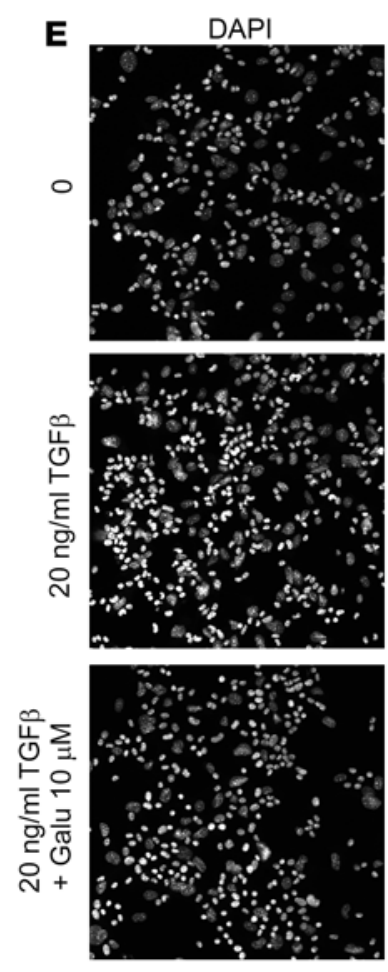

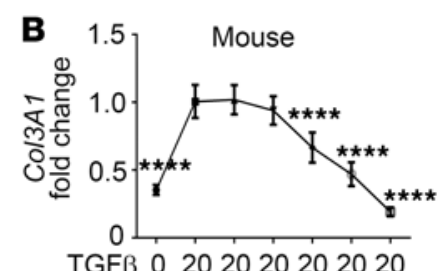

TGFß 0202020202020

Galu $0 \quad 0.25 .5 \quad 1 \quad 510$
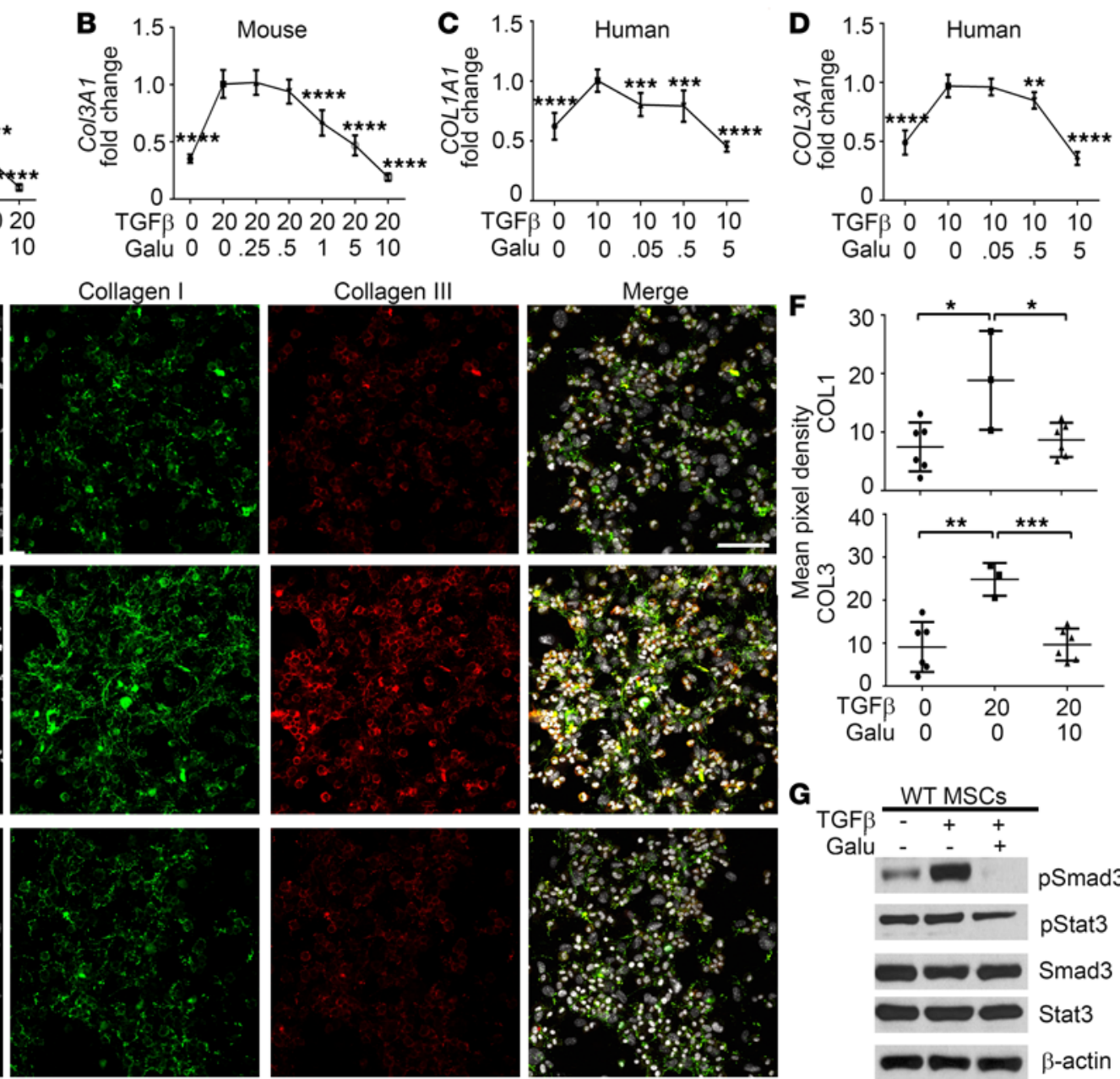

Figure 2. Galunisertib antagonizes the stimulatory effect of TCF- $\beta 1$ on collagen production by normal murine and human MSCs in vitro. (A and $B$ ) Mesenchymal stromal cells (MSCs) derived from MPL ${ }^{W T}$ mice $(n=6)$ were cultured in the presence of TGF- $\beta 1$ and different concentrations of galunisertib (Galu) in vitro for 72 hours, following which collagen I (Col1A1) and collagen III (Col3A1) mRNA levels were assessed by qRT-PCR with TATA sequence-binding protein (TBP), as reference gene (70). ${ }^{*} P<0.05,{ }^{* *} P<0.01,{ }^{* * *} P<0.0001$ by ANOVA, multiple comparisons using $20 \mathrm{ng} / \mathrm{ml}$ TGF- $\beta$ group as control. (C and D) Human COL1A1 and COL3A1 mRNA levels were measured by qRT-PCR in MSCs that were derived from normal human bone marrow $(n=3)$ and treated with human TGF- $\beta$ (hTCF- $\beta$ ) and galunisertib for 72 hours. ${ }^{* *} P<0.01,{ }^{* *} P<0.001,{ }^{* * *} P<0.0001$ by ANOVA, multiple comparisons using 10 ng/ $\mathrm{ml}$ hTCF- $\beta$ group as control. Results are representative of 3 independent experiments. (E) Representative immunofluorescence images showing collagen I (red), collagen III (green), and DAPI (white) in MSCs derived from MPL ${ }^{W T}$ mice with different treatments in vitro. A merged image is shown on the right. Scale bar: $75 \mu \mathrm{m}$. (F) Mean pixel intensity was acquired from the immunofluorescence images $(n=3-6)$ to quantify collagen I and collagen III deposition. ${ }^{*} P<0.05,{ }^{* *} P<0.01,{ }^{* * *} P<0.001$ by ANOVA, followed by Dunnett's multiple comparison test. All dot plots represent randomly taken images with means indicated. (G) Western blot showing p-Smad3 and p-STAT3 in MSCs derived from MPL ${ }^{W T}$ mice under indicated treatments, which represents 1 of 3 independent experiments conducted. See complete unedited blots in the supplemental material.

presses collagen production, MSCs from $M P L^{W 515 L}$-transplanted mice were treated with galunisertib in vitro. Galunisertib significantly decreased Col1A1 and Col3A1 production by these MSCs to levels that approached or were reduced compared with mice transplanted with $M P L^{W T}$-expressing virus (Figure 3, C and D). Collagen I and collagen III ECM deposition was also markedly diminished by galunisertib treatment, as shown by immunofluorescence staining (Figure $3, \mathrm{E}$ and $\mathrm{F}$ ), which is consistent with a role for TGF- $\beta$ in $\mathrm{MF}$ and suggests that galunisertib is a possible therapy for MF. Furthermore, p-Smad3 and p-STAT3 were suppressed by galunisertib in a dose-dependent manner in $M P L^{W 515 L}$ bone marrow-derived MSCs, suggesting that this pathway is susceptible to inhibition with this drug (Figure 3G). These findings indicate that TGF- $\beta$ blockade by the ALK5 inhibitor galunisertib may suppress the fibrotic phenotype of $M P L^{W 515 L}$-derived MSCs.

In vivo treatment with galunisertib significantly improves myelofibrosis in MPL W515L mice. Next, the antifibrotic effect of galunisertib was determined after in vivo treatment, as outlined in Figure 4A. MPL W515L mice were randomized to receive vehicle or galunisertib at $150 \mathrm{mg} / \mathrm{kg}$ or $300 \mathrm{mg} / \mathrm{kg}$ daily from day 12 
A

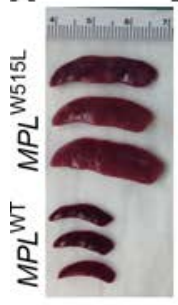

E

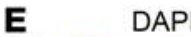

B

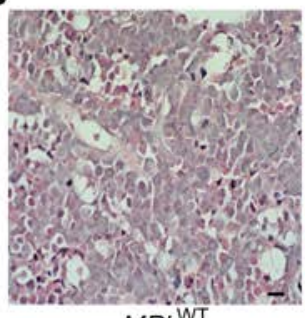

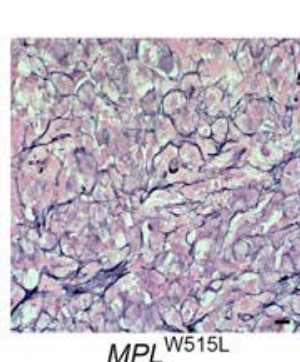

C

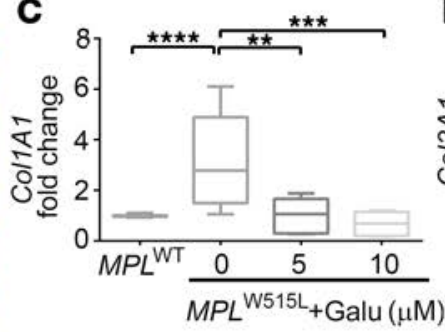

D

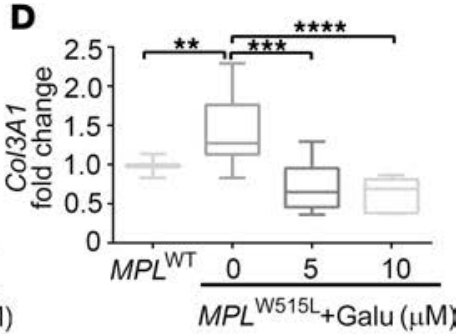

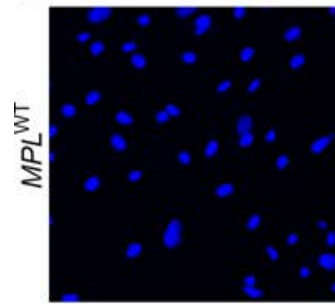
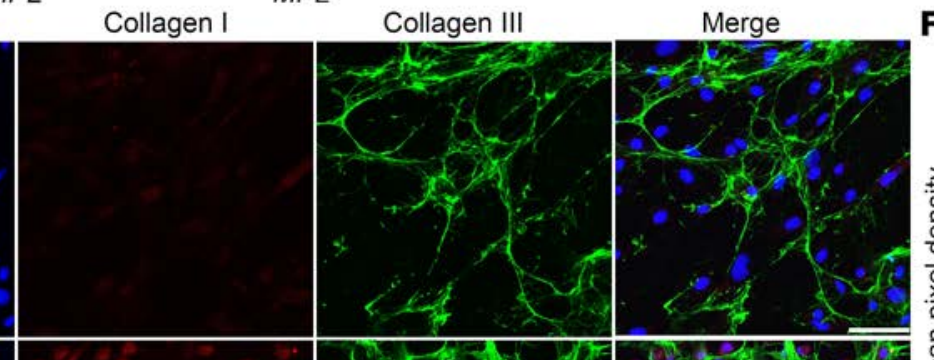

$\mathbf{F}$

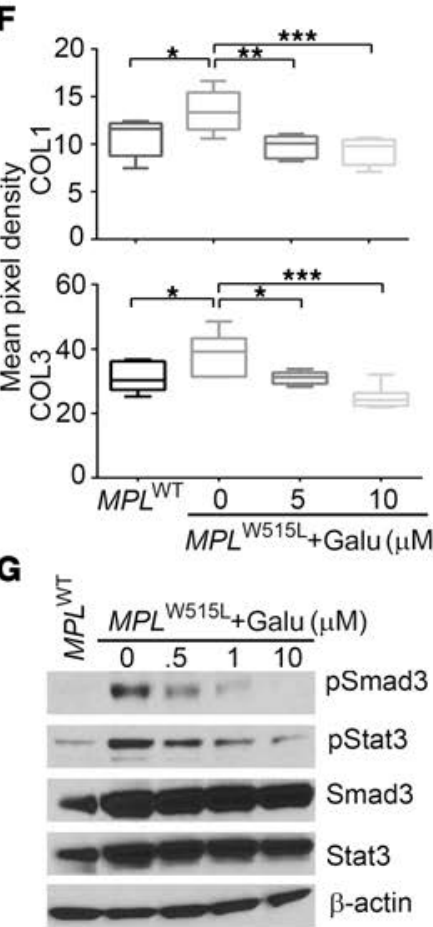

Figure 3. Galunisertib suppresses collagen production by MSCs derived from MPL ${ }^{\text {w515L }}$ mice in vitro. (A) Photographs of excised spleens from $M P L^{W T}$ ( $n=3$ ) and $M P L^{W 515 L}(n=3)$ mice to demonstrate the exemplary increase in size of MPL ${ }^{\text {W515L }}$ mice consistent with the presence of an myeloproliferative neoplasmlike (MPN-like) disease. (B) Reticulin staining was performed with bones isolated from MPL ${ }^{\text {WT }}$ and MPL ${ }^{\text {W515L }}$ mice. Scale bars: $10 \mu$ m. (C and D) Col1A1 and Col3A1 mRNA levels were assessed by qRT-PCR in mesenchymal stromal cells (MSCs) derived from MPL ${ }^{W T}$ and $M P L^{W 515 L}$ mice. Results are representative of 3 independent experiments. ${ }^{* *} P<0.01$, ${ }^{* * *} P<0.001$, ${ }^{* * *} P<0.0001$ by ANOVA, followed by Dunnett's multiple comparison test. (E) Representative immunofluorescence images of collagen I (red), collagen III (green), and DAPI (blue) in MSCs derived from MPL ${ }^{\text {w515L }}$ mice and treated with galunisertib (Galu) in vitro. A merged image is shown on the right. Scale bar: $75 \mu \mathrm{m}$. (F) Mean pixel fluorescent intensity was acquired for the images in $\mathbf{D}$ ( $n=5$ ) to compare collagen deposition. ${ }^{*} P<0.05,{ }^{* *} P<0.01,{ }^{* *} P<0.001$ by ANOVA, followed by Dunnett's multiple comparison test. In C, $\mathbf{D}$, F, and $\mathbf{G}$, graphs represent the mean and $95 \%$ confidence interval represented as a box-and-whisker blot of 5 individual mice or images per group. (G) Signaling pathways including $p-S m a d 3$ and p-STAT3 were detected by Western blot in MSCs. See complete unedited blots in the supplemental material.

after bone marrow transplantation for 14 days by oral administration. Representative flow cytometry plots show $\mathrm{GFP}^{+}$cells in mice transduced with $M P L^{W T}$ (Figure 4, B and C) and $M P L^{W 515 L}$ (Figure 4, D and $\mathrm{E}$ ) on day 0 and on day 14 after therapy. After treatment on day 14, MF was assessed by reticulin stain (Figure 4, F-H) and assigned a pathology score of 1 to 3 based on the European consensus grading scale used to assign fibrosis severity to patients with MF (34) (Figure 4I). Additionally, hydroxyproline quantification was used to quantify these results, as shown in Figure 4J. Compared with severe fibrosis in the vehicle-treated group, reduced reticulin staining was seen in $M P L^{W 515 L}$ mice receiving the ALK5 inhibitor (Figure 4, G and H). Fibrosis grade showed a reduced trend in mice treated with 150 $\mathrm{mg} / \mathrm{kg}$, but was significantly reduced in mice treated with $300 \mathrm{mg} / \mathrm{kg}$ galunisertib (Figure $4 \mathrm{C}$ ). Since hydroxyproline is a direct measure of the amount of collagen in tissues (35), hydroxyproline content in femurs was markedly reduced to near baseline levels in both the $150 \mathrm{mg} / \mathrm{kg}$ galunisertib and the $300 \mathrm{mg} / \mathrm{kg}$ galunisertib group compared with vehicle control-treated animals, providing quantitative assessment of decreased fibrosis with ALK5 inhibition (Figure 4J). 
A

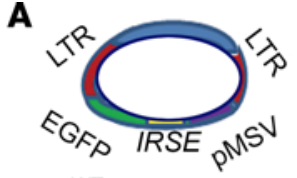

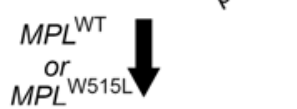

Infected Donor BM

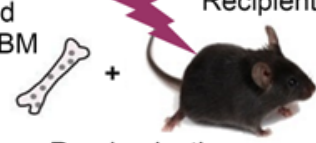

Randomization

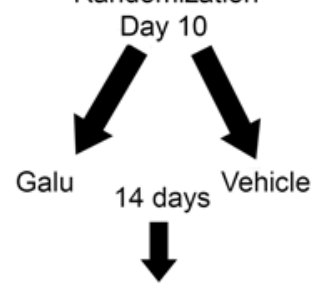

Assess Myelofibrosis

- Reticulin stain with pathology review and assignment of fibrosis score

- Hydroxyproline

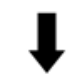

Establish MSC lines
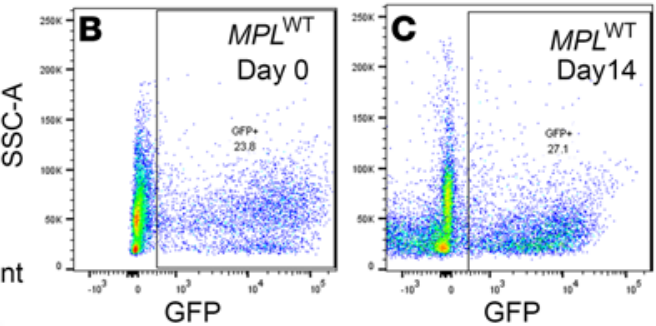

$\mathbf{G}^{\mathrm{G}}$
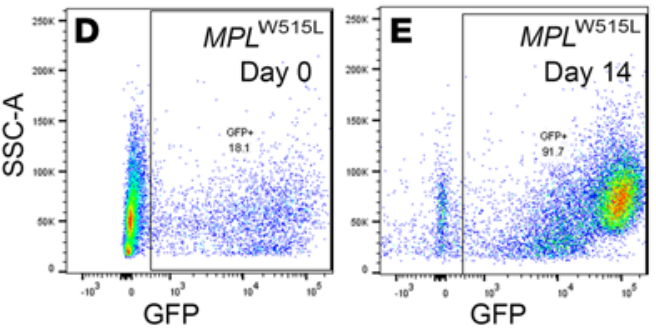

H
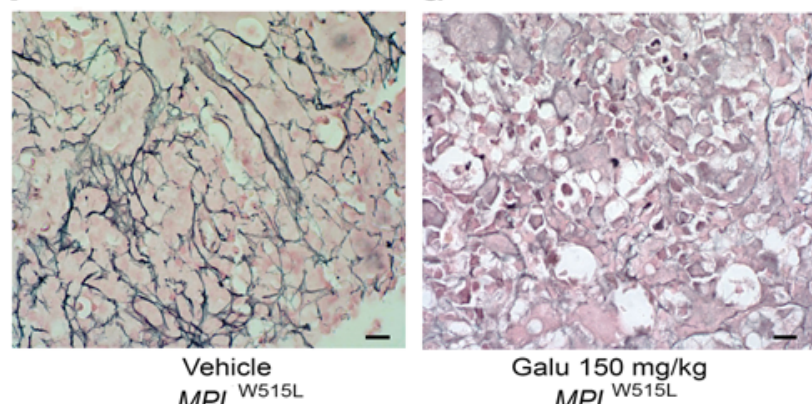
Galu $150 \mathrm{mg} / \mathrm{kg}$
$M P L^{\text {W515L }}$

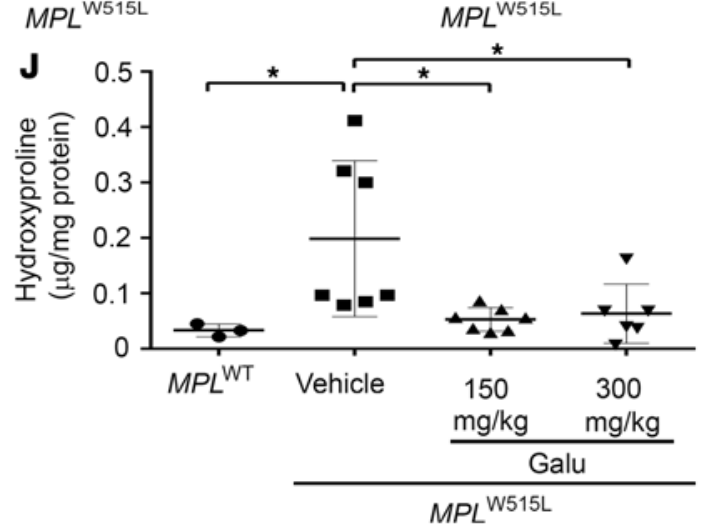

Figure 4. Galunisertib in vivo treatment significantly improves myelofibrosis of $M P L^{\text {w515L }}$ mice. (A) Diagram showing the procedures of the experiments. Bones isolated from MPL ${ }^{W 515 L}$ mice were randomized on day 10 to receive 1 of 2 doses of galunisertib (Galu) or vehicle control. Then, the mice were treated by oral gavage once daily for 14 days. (B-E) Representative flow cytometry staining of GFP+ retrovirally transduced bone marrow cells from $M P L^{W T}$ ( $\mathbf{B}$ and $\mathbf{C}$ ) and $M P L^{W 515 L}$ ( $\mathbf{D}$ and $\mathbf{E}$ ) on day 0 (representative mouse randomly selected and sacrificed) and on day 14 after treatment when all mice were sacrificed and further examined for pathological changes and for hydroxyproline quantification. Reticulin staining was then performed to evaluate fibrosis. Representative images of the reticulin stains are shown after treatment with vehicle (F), $150 \mathrm{mg} / \mathrm{kg}$ galunisertib (G), and $300 \mathrm{mg} /$ $\mathrm{kg}$ galunisertib $\mathbf{( H )}$ to assess myelofibrosis in MPL ${ }^{\text {W515L }}$ mice. Scale bars: $10 \mu \mathrm{m}$. (I) Fibrosis was scored according to European consensus on grading myelofibrosis $(n=3)$. ${ }^{*}<0.05$ by ANOVA, followed by Dunnett's multiple comparison test. (J) Hydroxyproline amount was determined in the bones to quantify myelofibrosis in MPL ${ }^{W T}$-transduced mouse bone marrow, vehicle $(n=7), 150 \mathrm{mg} / \mathrm{kg}$ galunisertib $(n=7)$, and $300 \mathrm{mg} / \mathrm{kg}$ galunisertib ( $\left.n=6\right)$ groups. ${ }^{*} P<0.05$ by ANOVA, followed by Dunnett's multiple comparison test.

Galunisertib in vivo treatment suppresses the fibrotic phenotype of the MSCs from MPL W515L mice. Based on the collective findings, the effect of galunisertib treatment in vivo was assessed on abnormal MSCs. Cultured MSCs were established from $M P L^{W 515 L}$ mice after galunisertib or vehicle in vivo treatment with the $300 \mathrm{mg} / \mathrm{kg}$ dose (Figure 5A). The morphological appearance of profibrotic MSCs has previously been shown to adopt a fattened shape relative to normal MSCs (14). Compared with $M P L^{W T}$-transduced mice (Figure 5B), MSCs from $M P L^{W 515 L}$ mice displayed an abnormal morphological shape (Figure 5C), which was reversed by galunisertib treatment (Figure 5D). MSCs from galunisertib-treated mice produced significantly less Col1A1 and Col3A1 mRNA compared with those from vehicle-treated mice measured by qRT-PCR (Figure 5E), and both p-Smad3 and p-STAT3 were downregulated in the MSCs from galunisertib-treated mice compared with vehicle-treated mice (Figure 5F). These results suggest that galunisertib suppresses the fibrotic phenotype associated with malignant hematopoiesis by blocking the TGF- $\beta$ signaling pathway activated in MSCs. Subsequent decrease in collagen production is consistent with the mechanism for improved MF in galunisertib-treated MPL W515L transplanted mice.

In vivo treatment with galunisertib does not significantly change blood counts and spleen size in MPL ${ }^{W 515 L}$-transduced mice. There have been concerns that TGF- $\beta$ suppression may promote myeloid proliferation, thus 
A

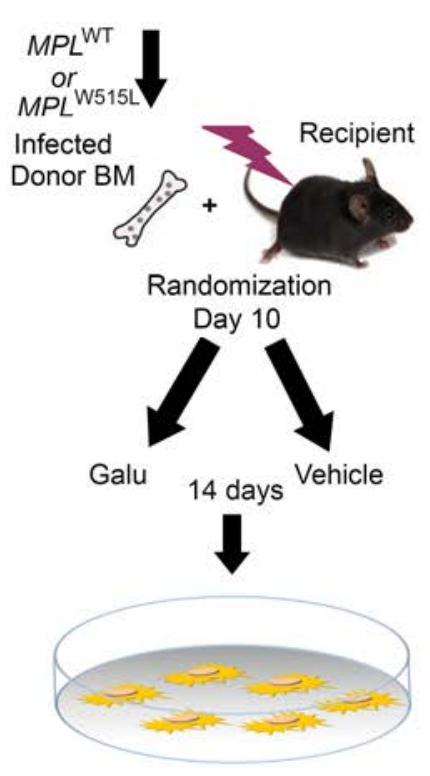

Cultured MSCs
B
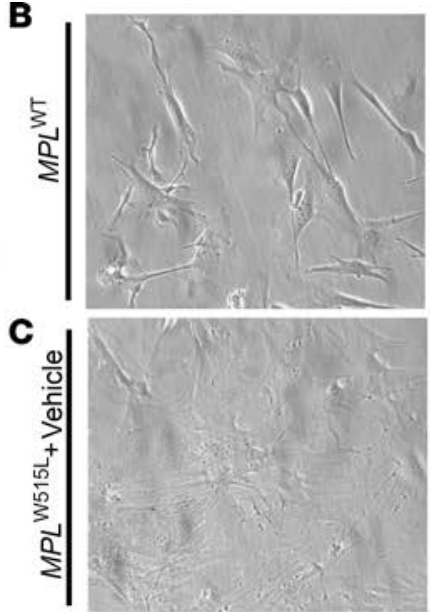

D

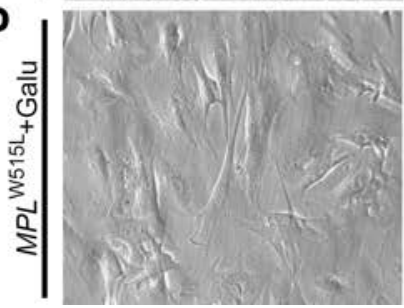

E
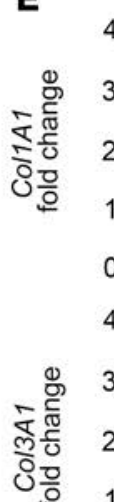

$\mathbf{F}$

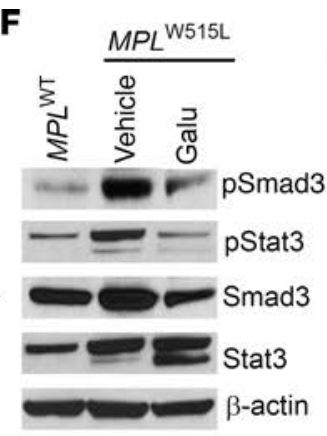

Figure 5. Galunisertib in vivo treatment reverses the fibrotic phenotype of the MSCs in $\mathbf{M P L}{ }^{\text {w515L }}$ mice. (A) Diagram showing the procedures of the experiments. After randomization on either day 10 or day 12, groups of mice were treated for 14 days with of vehicle or galunisertib (Galu) in vivo. Mesenchymal stromal cells (MSCs) were then isolated from MPL ${ }^{\text {W515L }}$ mice and cultured for collagen production analysis. (B-D) Representative images of MSCs from $M P L^{W T}(\mathbf{B})$ and $M P L^{W 515 L}$ mice treated with vehicle (C) or $300 \mathrm{mg} / \mathrm{kg}$ galunisertib (D) are shown. Original magnification, $\times 250$. (E) Col1A1 and Col3A1 mRNA production was measured by qRT-PCR relative to TATA sequence-binding protein (TBP) in the MSCs $(n=6)$. ${ }^{*} P<0.01$, ${ }^{* * *} P<0.001$ by ANOVA, followed by Dunnett's multiple comparison test. (F) Signaling pathways in the MSCs were detected by Western blot after galunisertib in vivo treatment. See complete unedited blots in the supplemental material.

increasing disease burden. Therefore, we evaluated tumor burden in $M P L^{W 515 L}$ mice after galunisertib treatment. Peripheral blood cell counts were similar in vehicle- and galunisertib-treated animals (Figure 6, A-C). Although not significant, mice receiving galunisertib showed some inconsistency, but slightly decreased spleen weights (Figure 6, D and E) compared with those receiving vehicle. No difference was observed in liver weight among vehicle- or galunisertib-treated mice (Figure $6 \mathrm{~F}$ ). Bone marrow populations were also assessed using flow cytometry. The percentages of $\mathrm{GFP}^{+}$cells (Figure 6G), myeloid cells (CD11b+, Figure $6 \mathrm{H}$ ), $\mathrm{GFP}^{+} \mathrm{CD}_{4} 1^{+}$megakaryocytes (Figure $6 \mathrm{I}$ ), and $\mathrm{GFP}^{+} \mathrm{CD} 71^{+}$erythrocytes (Figure $6 \mathrm{~J}$ ) show no significant differences in response to galunisertib treatment. These data support the safety of TGF- $\beta$ blockade in an aggressive MPN animal model driven by the $M P L^{W 515 L}$ mutation.

$M F$ in JAK2 $2^{V 617 F}$ transgenic mice is significantly reduced by galunisertib in vivo treatment. Further evaluation of the therapeutic effect of galunisertib was conducted using $J A K 2^{V 617 F}$ transgenic mice (36). These mice display high levels of peripheral blood cell counts at early stages, and begin to develop MF at 20 weeks old and by 30 weeks present with severe splenomegaly and progressive MF (36). Transcriptome analysis of total RNAs from whole femurs revealed that $T g f b 1$ and several TGF- $\beta$-induced genes were overexpressed in 56-week-old $J A K 2^{V 617}$ mice with severe MF (Figure 7, A-C). The reason for using femur RNAs for the analysis is that it was difficult to collect bone marrow cells from mice with severe MF. Nonetheless, qRT-PCR analysis confirmed the overexpression of $T g f b 1$ in both femur and bone marrow cells (Figure 7B). The data suggest that TGF- $\beta 1$ may also contribute to MF in $J A K 2^{V 617}$ transgenic mice. Since these mice show relatively chronic disease progression compared with $M P L^{W 515 L}$ mice, a lower dose of galunisertib was administered orally for an extended period of time. Galunisertib was orally administered to 30-week-old $J A K 2^{V 617 F}$ transgenic mice at $50 \mathrm{mg} / \mathrm{kg}$ daily for 4 weeks. Bone marrow reticulin stains demonstrated improvement of MF in the galunisertib-treated group compared with vehicle-treated mice (Figure 7C). Hydroxyproline content was also quantified from the femurs of the mice, confirming that collagen production is decreased by drug treatment $(P=0.005)$ (Figure 7D). Note that the drug treatment did not cause changes in blood cell counts (Figure 7, E-G) but showed significantly reduced spleen size $(P=0.04)$ (Figure $7 \mathrm{H})$. 

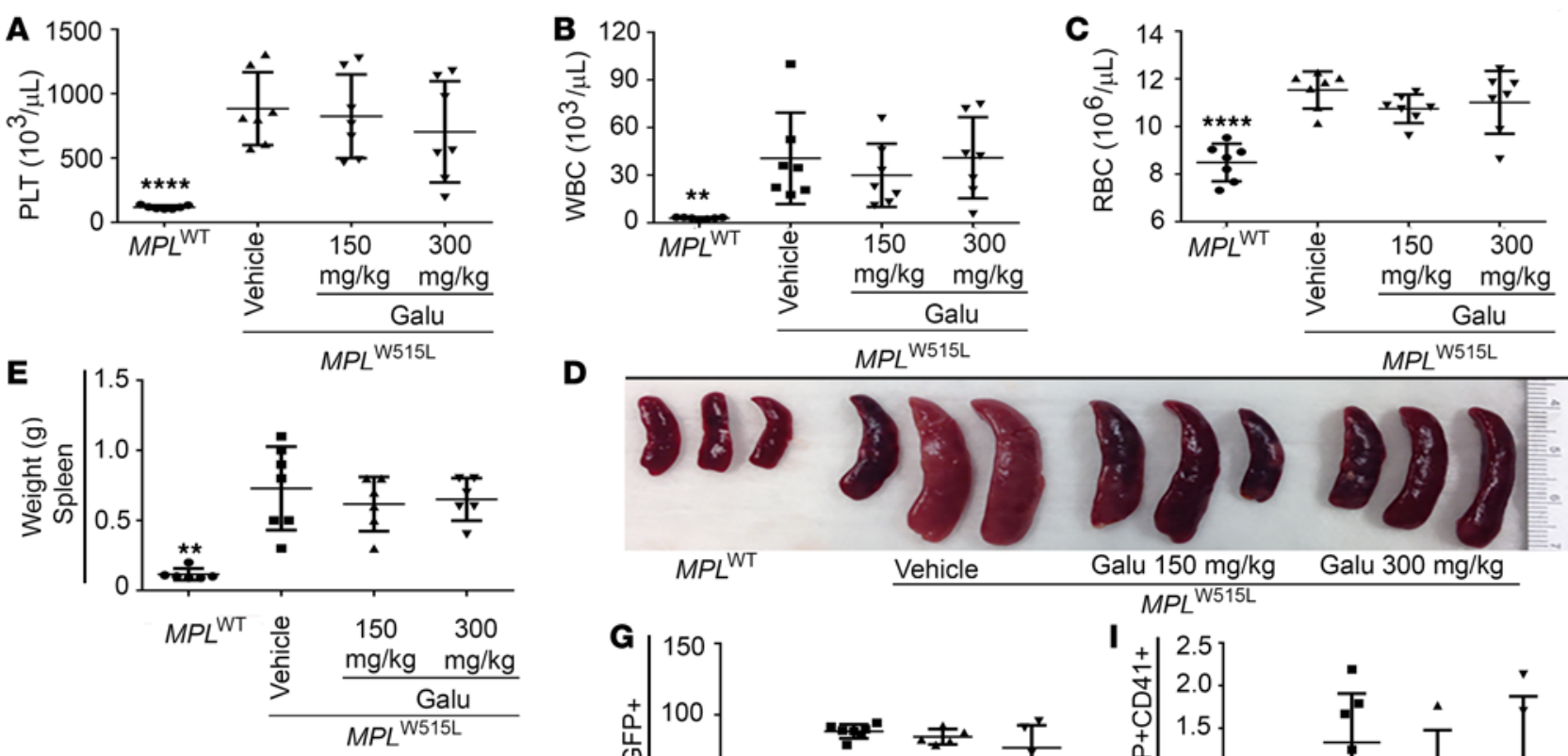

D
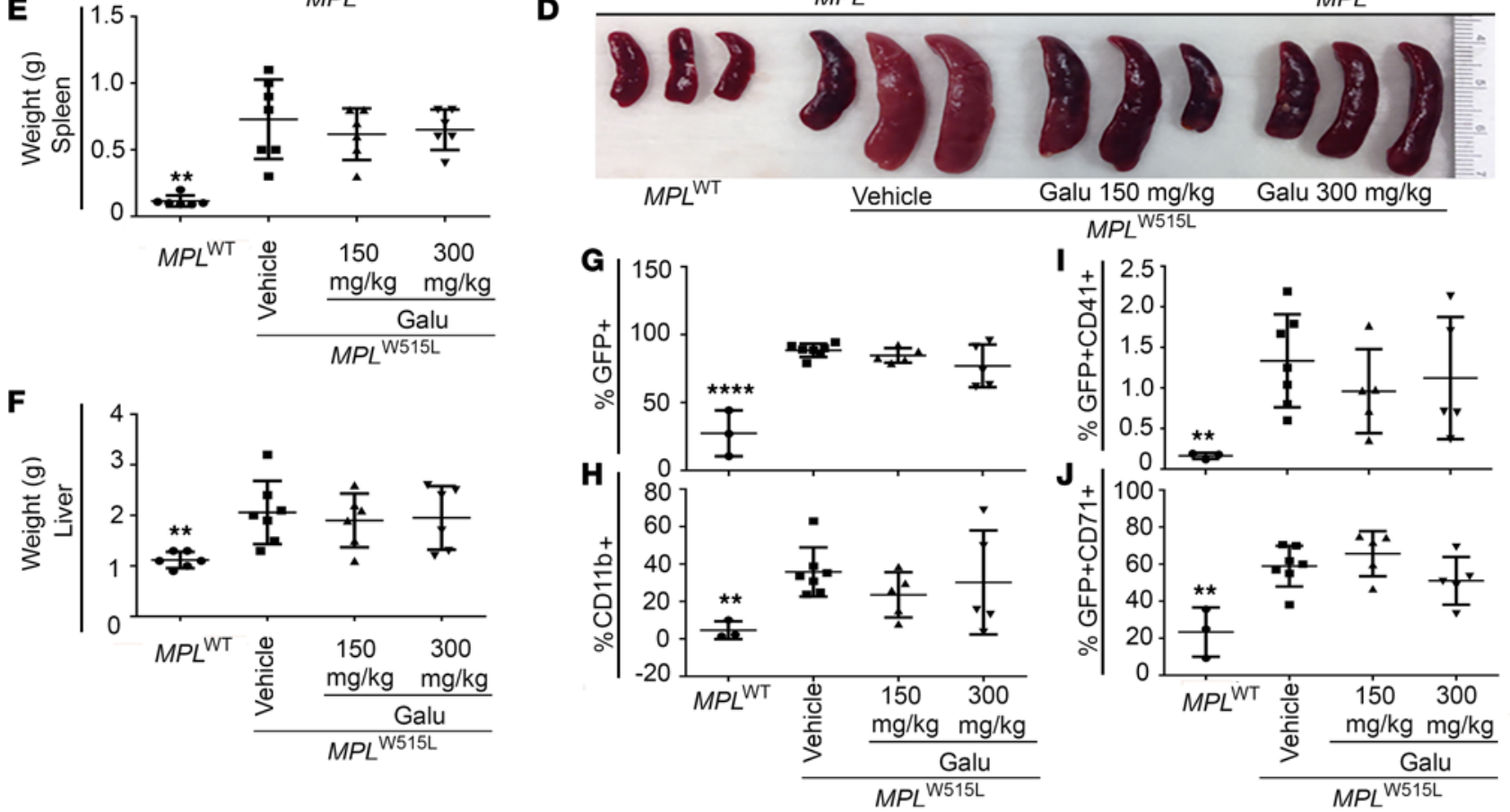

Figure 6. In vivo treatment with galunisertib does not increase tumor burden of MPL $^{\text {w515L }}$ mice. (A-C) Peripheral blood cell counts of $M P L^{\text {W515L }}$ mice after 14 days treatment with either vehicle or $150 \mathrm{mg} / \mathrm{kg}$ or $300 \mathrm{mg} / \mathrm{kg}$ galunisertib (Galu) in vivo. Values of $M P L^{W T}$ mice served as normal control. $n=7$. Results are representative of 3 independent experiments. ${ }^{* *} P<0.01$, ${ }^{* * *} P<0.0001$ by ANOVA, followed by Dunnett's multiple comparison test using vehicle group as control. PLT, platelets. (D) Photographs of excised spleens from MPL ${ }^{W T}$ and $M P L^{W 515 L}$ mice in $\mathbf{A}-\mathbf{C}$. (E) Spleens and (F) livers were isolated from the mice and weights were compared $(n=6) .{ }^{* *} P<0.01$ by ANOVA, followed by Dunnett's multiple comparison test using vehicle group as control. Flow cytometry for bone marrow cell populations was conducted after gating on viable cells (using DAPI) including percentages of (C) GFP+, (H) CD11 $\mathbf{b}^{+}$, (I) $\mathrm{GFP}^{+} \mathrm{CD} 41^{+}$, and $(\mathrm{J}) \mathrm{GFP}+\mathrm{CD} 71^{+}$. Percentages were assessed in 5 individual mice and repeated in 3 independent experiments (data points represent individual mice with means indicated). ${ }^{* *} P<0.01,{ }^{* * *} P<0.0001$ by ANOVA, followed by Dunnett's multiple comparison test using vehicle group as control.

\section{Discussion}

TGF- $\beta$ is a well-established fibrotic cytokine that plays a vital role in the pathophysiology of MF as well as other fibrotic diseases $(15,17,19-21,37-42)$. Elevated levels of the TGF- $\beta 1$ isoform were detected in plasma and bone marrow serum in MF mouse models. Overexpression of thrombopoietin generates typical features of MF in WT mice, but this phenotype is prevented by the homozygous genetic deletion of TGF- $\beta(16,17,43)$. However, targeting TGF- $\beta$ for the treatment of MF has not been accomplished clinically owing to the lack of clinical inhibitors of this pathway. In addition, TGF- $\beta 1-$ mediated changes to the bone marrow niche remain to be fully elucidated. In this study, we first show that normal MSCs produce increased ECM collagen components after TGF- $\beta 1$ treatment, mimicking the fibrotic features of MSCs derived from $M P L^{W 515 L}$ mice. The TGF- $\beta 1$ isoform is also overexpressed in both MF patients and $M P L^{W 515 L}$ mouse samples, further reinforcing that TGF- $\beta 1$ is responsible for reprogramming the MSCs present in the bone marrow niche, which induces them to adopt the fibrotic phenotype. Most importantly, we show the efficacy of a clinical inhibitor of ALK5 in mouse models of MF.

Our results further the understanding of the bone marrow niche in MF. MSCs are multipotent stromal cells that are critical components of bone marrow niche that regulate proliferation, differentiation, and 


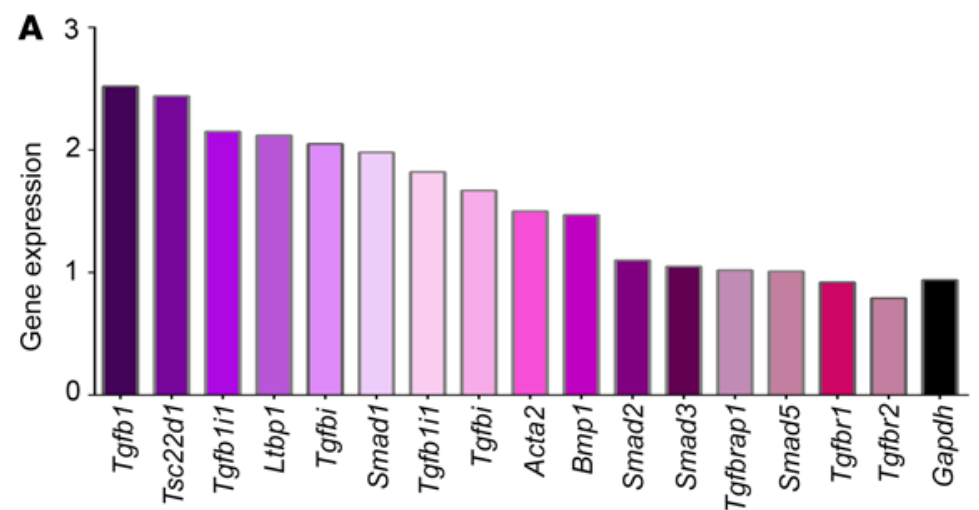

C

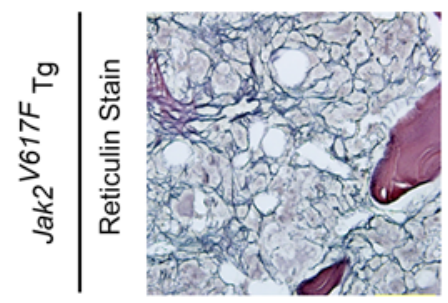

Vehicle

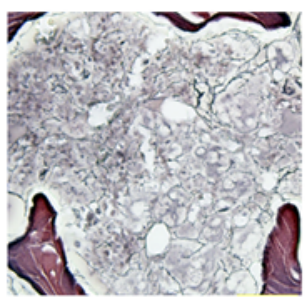

Galu $50 \mathrm{mg} / \mathrm{kg}$
B

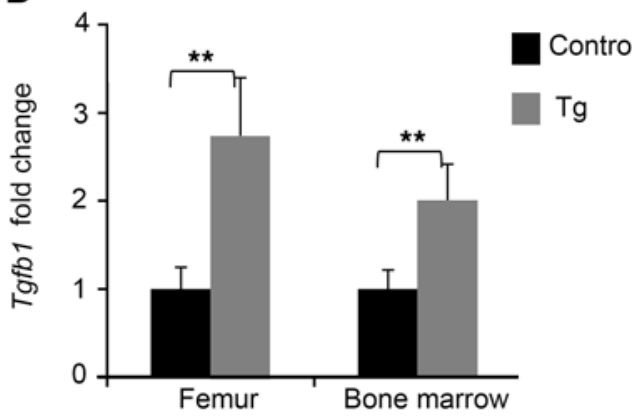

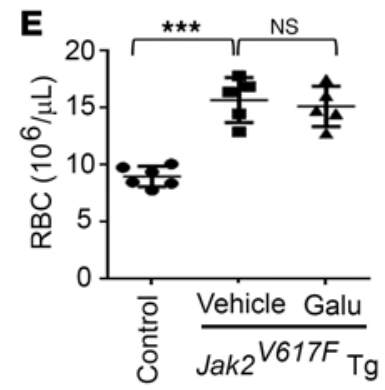
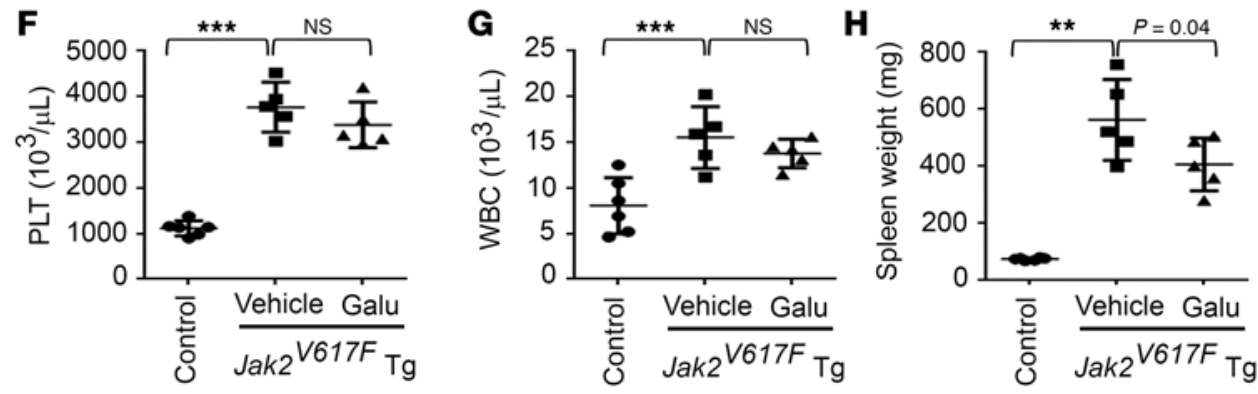

Figure 7. TGF- $\beta$ is elevated in JAK2 ${ }^{\mathrm{v617F}}$ transgenic mice and galunisertib treatment markedly reduces myelofibrosis. (A) cDNA microarray was performed with total RNAs from femurs of 56-week-old JAK2 ${ }^{\mathrm{V} 617 F}$ transgenic and control mice. Changes in expression levels of 16 probe sets representing 13 distinct genes are shown that are involved in the TCF- $\beta$ pathway together with the housekeeping gene Gapdh. (B) Real-time PCR confirmed elevation of Tgfb1 expression in femurs from 56-week-old mice and bone marrow cells from 30-week-old mice (4 mice used in each group). ${ }^{* *} P<0.01$ by unpaired 2 -sided Student's $t$ test, control vs. transgenic (Tg). (C-H) JAK2 $2^{\mathrm{V} 677}$ transgenic mice (30-week-old males, 5 in each group) were treated through oral gavage with vehicle control or galunisertib (Galu) at a daily dose of $50 \mathrm{mg} / \mathrm{kg}$ for 4 weeks. Data represent images of reticulin staining of femurs from vehicle- and drug-treated JAK2 ${ }^{\mathrm{V} 677 \mathrm{~F}}$ transgenic mice $(\mathbf{C}$; original magnification, $\times 200)$, quantification of fibrosis by determining hydroxyproline contents in femurs (D), and blood cell counts $(\mathbf{E}-\mathbf{C})$ together with spleen weights $(\mathbf{H})$. Untreated nontransgenic mice of comparable ages are provided for comparison $(n=6)$. PLT, platelets. Error bars indicate the mean $\pm \mathrm{SD}$. ${ }^{* *} P<0.01,{ }^{* *} P<0.001$ by ANOVA, followed by Dunnett's multiple comparison test. n.s., not significant.

homing of hematopoietic stem cells (HSCs). They possess self-renewal and immunomodulatory capacities and could differentiate into osteoblasts, adipocytes, and chondrocytes under specific conditions (44-46). Evidence indicates that neoplastic HSCs lead to profound modification of the bone marrow niche, which in turn affects the proliferation and trafficking of HSCs in $\operatorname{MF}(6,9,11,47-49)$. Although the mechanism for these profound changes in the bone marrow niche are poorly defined, recent studies reported that MSCs in MPN patients have elevated fibronectin levels and exhibit both genetic and functional aberrations $(50,51)$. Martinaud et al. showed that MF-MSCs have increased osteogenic potential contributing to MF development (52). Our previous results show that cultured MSCs do not originate from HSCs and do not have $M P L^{W 515 L}$ mutations (53). In patient-derived xenografts, Verstovsek et al. showed that fibrosis may also be caused by fibrocytes which are morphologically distinct from MSCs and are derived directly from a CD14 ${ }^{+}$monocyte mutant precursor cell population (54). In our study based on the finding that MSCs from MF mice produced elevated collagen I and collagen III, which accounted for osteosclerosis and fibrillar ECM, respectively, we further confirm the role of TGF- $\beta 1$ in MSC modulation and bone marrow niche remodeling in MF. Although malignant fibrocytes were not studied here, traditional MSCs from recipient mice are indirectly modified by HSCs and are notable contributors to fibrosis. 
Based on these observations, the effect of TGF- $\beta$ blockade with galunisertib was further tested in MF mouse models. It has been challenging to develop TGF- $\beta$ inhibitors for clinical use as they can have cardiac-adverse effects, can affect bone development, and also alter inflammatory responses in the skin or gut of rats and dogs. It has also been shown that blocking TGF- $\beta$ signaling can cause chronic inflammation in certain models (26). Significant improvement in the severity of fibrosis was observed in 2 different models of MPN driven by $M P L^{W 515 L}$ and $J A K 2^{V 617 F}$. The observation that ALK5 inhibition reverses the abnormal morphology and fibrotic characteristics of MSCs both in vitro and in vivo provides a mechanistic explanation for the rational development of TGF- $\beta$ pathway inhibitors for the treatment of MF. Galunisertib is an ALK5 inhibitor that has been shown to be safe in human studies and is a potential therapeutic agent in MF owing to good specificity, tolerability, and oral administration (28). The ALK5/Smad3 pathway is considered to be critical in the profibrotic activities mediated by TGF- $\beta 1(55,56)$. Loss of Smad3 interrupts TGF- $\beta 1-$ mediated induction of collagen genes (57). Smad3 null mice displayed attenuated fibrosis in several experimental models such as bleomycin-induced pulmonary fibrosis (58), irradiation-induced dermal fibrosis (59), and cardiac fibrosis $(60,61)$. In our findings, we observed elevated Smad3 signaling in $M P L^{W 515 L}$-derived MSCs and normal MSCs treated with TGF- $\beta 1$, both of which were markedly suppressed by galunisertib. Collectively, galunisertib treatment effectively inhibits the intrinsically elevated ALK5/ Smad3 pathway, leading to a diminution in collagen production by abnormal MSCs. Interestingly, we also observed decreased p-STAT3 by TGF- $\beta$ blockade, which may result from the modulation of interacting pathways $(62,63)$ important for regulation of collagen production and inflammation related to IL-6 (64).

Detecting TGF- $\beta$ protein levels in human blood samples has been problematic owing to patient heterogeneity, issues with sample collection, and the need for platelet depletion for adequate estimation of levels. Thus, we analyzed TGF- $\beta 1$ levels at the expression level in purified neutrophils and found that PMF patients specifically had significant overexpression of this cytokine. Previous efforts to test TGF- $\beta 1$ suppression in MF (65) were conducted by Shehata et al. They found that TGF- $\beta 1$ enhances collagen production by fibroblasts, which is reduced by TGF- $\beta 1-$ neutralizing antibodies in vitro (43). Regarding in vivo treatment, there has been 1 clinical trial with anti-TGF- $\beta$ antibody (GC1008) reported, which enrolled 3 subjects before it was terminated. However, transfusion independence was observed in 1 of the 3 subjects and 12 cycles of treatment did not induce disease progression or leukemic transformation, which was consistent with our findings in this study (66). Furthermore, there are data suggesting that stroma-derived cytokines might protect $J A K 2^{V 617 F}$-mutated cells against JAK2 inhibitor therapy (67). This highlights the importance of targeting the interaction between neoplastic HSCs and the bone marrow niche to rescue the abnormal niche and reverse stroma-mediated protection of the malignant clone.

Collectively, in this study using 2 distinct MF mouse models, the profibrotic role of TGF- $\beta 1$ was confirmed and a direct role in modulating MSC-derived ECM was established. Therapeutic effects of TGF- $\beta$ pathway blockade appears to reverse, not only prevent, fibrosis severity in the $J A K 2^{V 617 F}$ MPN model, which is currently observed only after HSC transplantation. Therapies targeting the interplay between HSCs and MSCs in MF may improve the clinical outcome of MF patients.

\section{Methods}

Animal experiments. The $M P L^{W 515 L}$ transplantation model of MPN was established using procedures as described previously with minor modifications (68). Bone marrow cells were harvested from C57BL/6 donor mice 7 days after 5 -fluorouracil injection $(150 \mathrm{mg} / \mathrm{kg})$. Cells were then treated with red blood cell lysis buffer and cultured overnight in transplantation medium (RPMI $+10 \% \mathrm{FBS}+6 \mathrm{ng} / \mathrm{ml} \mathrm{IL}-3$, $10 \mathrm{ng} / \mathrm{ml} \mathrm{IL}-6$, and $10 \mathrm{ng} / \mathrm{ml}$ stem-cell factor) at $37^{\circ} \mathrm{C}$ and $5 \% \mathrm{CO}_{2}$. The next day cells were transduced with recombinant retroviruses overexpressing either $M P L^{W 515 L}$ or $M P L^{W T}$ by spin infection at $1,140 \mathrm{~g}$ for 90 minutes at $30^{\circ} \mathrm{C}$. The spin infection was repeated 24 hours later. Cells were then resuspended in PBS and injected into tail veins of lethally irradiated $(2 \times 450 \mathrm{cGy})$ C57BL/ 6 recipient mice at $0.8 \times 10^{6}$ to $1.0 \times 10^{6}$ cells/mouse. Viral constructs used included MSCV-human-MPL ${ }^{W 515 L}$-GFP and MSCV-human- $M P L^{W T}$-GFP (68). Retroviral vectors were obtained from Ross Levine at the Memorial Sloan-Kettering Cancer Center. Recipient mice receiving $M P L^{W 515 L}$-transduced bone marrow developed leukocytosis, thrombocytosis, and MF in 2 to 3 weeks. In the interest of brevity, mice transplanted with $M P L^{W 515 L_{-}}$or $M P L^{W T}$-transduced bone marrow cells will be referred to as $M P L^{W 515 L}$ and $M P L^{W T}$ mice, respectively. For drug studies, $M P L^{W 515 L}$ mice were randomized to the vehicle or treatment group and peripheral blood cell counts among groups were comparable at the starting point. $M P L^{W T}$ mice served as 
nontumor controls. Vehicle (NaCMC/SLS/PVP/antifoam solution) or galunisertib was administered to $M P L^{W 515 L}$ mice by oral gavage daily from day 12 after bone marrow transplantation for 14 consecutive days. Stable homozygous $J A K 2^{V 617 F}$ transgenic mice were generated using a vav gene promoter, as previously described (36). The transgenic mice exhibited a stable phenotype that closely resembled PV at an early stage and developed MF as they aged, exhibiting a relatively chronic disease status. Thus, a lower dose of galunisertib at $50 \mathrm{mg} / \mathrm{kg}$ was administered to 30 -week-old mice orally once per day for 4 weeks after onset of fibrosis. Mice were sacrificed at the end of treatment and organs were harvested for analysis. Spleens were homogenized through a $100-\mu \mathrm{m}$ mesh, and splenocytes were frozen in FBS with $10 \%$ DMSO until sorting.

MSC isolation and culture. MSC cultures were established as previously described by adhesion to plastic $(14,69)$. Cells were cultured in $\alpha$ MEM (Life Technologies) supplemented with $10 \% \mathrm{FBS}, 100 \mathrm{U} / \mathrm{ml}$ penicillin, and $100 \mathrm{U} / \mathrm{ml}$ streptomycin under hypoxic conditions $\left(5 \% \mathrm{CO}_{2}, 93 \%\right.$ nitrogen, and $\left.2 \% \mathrm{O}_{2}\right)$ at $37^{\circ} \mathrm{C}$. Bone marrow aspirates from healthy human volunteers were obtained commercially (Lonza) and MSCs were established using similar methods. Culture medium was replaced at least once per week and cells were trypsinized and passaged when $90 \%$ confluence was reached. Cells from the second or third passages were used for experiments since there was no evidence of viral infection or hematopoietic contamination at this time (data not shown).

Virus production. For virus production, 293T cells $\left(2.6 \times 10^{6}\right)$ were seeded in a 10-cm dish the day before transfection and then transfected with MSCV-human- $M P L^{W 515 L}-\mathrm{GFP}$ or MSCV-human- $M P L^{W T}$-GFP plasmids together with envelope plasmids and $2.5 \mathrm{M} \mathrm{CaCl}_{2}$. Forty-eight hours after transfection, viral supernatant was collected and frozen until use.

Human MPN expression data. Expression patterns of various TGF ligands in human MPN neutrophil samples were obtained from a publically available gene expression cohort of samples representing PV, ET, and PMF (27).

qRT-PCR analysis. Total RNAs were extracted from cultured MSCs using an RNeasy Micro Kit (Qiagen), and converted into cDNA using iScript cDNA synthesis kit (Bio-Rad) according to the manufacturers' indications. cDNA was added to Taqman PCR mix (Life Technologies) in a final volume of $25 \mu 1$ containing forward and reverse primers. All primers were purchased from Life Technologies (Col1a1 Mm00801666 g1, Col3a1 Mm01254476_m1, Tgfß1 Mm01178820_m1, TATA-binding protein (TBP) Mm00446971_m1). Amplification cycles $(n=40)$ were performed on a 7900HT Real-time PCR System (Applied Biosystems). Data were normalized to the reference gene $T B P$ by a relative quantification using the $\triangle \triangle \mathrm{Ct}$ method.

Total RNA from sorted splenocytes was isolated using an RNeasy Micro Kit and reverse transcribed with the SuperScript III First-Strand Synthesis System (Invitrogen). Multiplex qPCR was performed with TaqMan hydrolysis probes for TgfB1 (Mm01178820_m1, FAM) and glyceraldehyde-3-phosphate dehydrogenase (Gapdh, Mm99999915_g1, VIC, primer limited) in TaqMan Multiplex Master Mix (all Life Technologies) on a CFX 96 touch thermocycler (Bio-Rad) in 45 amplification cycles. TGF $\beta 1$ levels were normalized to the reference gene Gapdh by a relative quantification using the $\Delta \Delta \mathrm{Ct}$ method.

cDNA microarray analysis. Total RNAs were isolated from bone marrow cells or whole femurs using the RNeasy Plus Mini Kit (Qiagen). The Illumina mouse ref-8 microarray was employed for analysis of gene expression in whole femurs from 56-week-old control and JAK2V617F transgenic mice using the core facility at the Oklahoma Medical Research Foundation. The data were normalized by using MATLAB and analyzed by using BRB ArrayTools. For real-time PCR confirmation, single-strand cDNAs were synthesized with equal amounts of total RNAs using the QuantiTect reverse transcription kit (Qiagen). Real-time PCR was performed with iQ SYBR Green Supermix (Bio-Rad) and TgfB1 primers 5'-GCGCTTGCAGAGATTAAAATCAA and 5'-GTAACGCCAGGAATTGTTGCTATA. Gapdh served as control as previously described (36).

Immunofluorescence staining. MSCs were cultured in Lab-Tek chamber slides (Nalgene Nunc) for 3 days to allow collagen production and formation of fibrillar structures. Polychromatic collagen stains were performed with antibodies against collagen types I (Abcam, ab6308) and III (Abcam, ab7778). Secondary stains were conducted using Alexa Fluor $647 \mathrm{~F}\left(\mathrm{ab}^{\prime}\right)_{2}$ donkey anti-mouse and Alexa Fluor $488 \mathrm{~F}\left(\mathrm{ab}^{\prime}\right)_{2}$ donkey anti-rabbit (Jackson ImmunoResearch Laboratories). Vectashield mounting medium containing DAPI (Vector Laboratories) was used to stain nuclei. To visualize the fibrillar structure, a Leica DMI6000 inverted microscope, TCS SP5 confocal scanner, and a 20×/0.7NA Plan Apochromat oil immersion objective (Leica Microsystems) were used. LAS AF lite version 2.6 was used to analyze fluorescence images and mean pixel intensity was calculated for each field. 
Western blot. MSCs were lysed in RIPA buffer (Sigma-Aldrich) supplemented with protease inhibitors (Roche) and phosphatase inhibitors (Sigma-Aldrich, P5726). Proteins were separated by SDS-PAGE and transferred onto a nitrocellulose membrane. Membranes were blotted with antibodies detecting p-Smad3 (Abcam, ab52903), Smad3 (Abcam, ab40854), p-STAT3 (Cell Signaling Technology, 9145) and STAT3 (Cell Signaling Technology, 9139).

Histology. Femurs were fixed in 10\% neutral-buffered formalin, decalcified in 5\% nitric acid, and embedded in paraffin. Sections $(2-4 \mu \mathrm{m})$ were mounted for Gomori's silver impregnation (reticulin). MF was scored according to European consensus grading scale (34).

Hydroxyproline quantification. Hydroxyproline assays were performed based on an established protocol (35). After fixation and decalcification, bone marrow tissues were dissected from the bones under a stereo microscope and hydrolyzed with $6 \mathrm{M} \mathrm{HCl}$ at $120^{\circ} \mathrm{C}$ for 3 hours. The hydrolysates were then dried in a speed vacuum concentrator and oxidized with chloramine $\mathrm{T}$ for 5 minutes at room temperature. Ehrlich's reagent was then added and incubated for 90 minutes at $60^{\circ} \mathrm{C}$. The absorbance was then measured at $560 \mathrm{~nm}$ using a spectrophotometer. Standard hydroxyproline was measured in the same way and hydroxyproline contents in the samples were calculated based on standard curves and normalized to total protein concentrations, which were determined by measuring $\mathrm{OD}_{280 \mathrm{~nm}}$ and $O D_{260 \mathrm{~nm}}$ of the hydrolysates.

Flow cytometry. Single-cell suspensions of bone marrow cells were prepared by resuspending the cells in PBS with 1\% BSA (Sigma-Aldrich) and 2 mM EDTA (Life Technologies). Surface marker staining was performed using the following antibodies: APC-CD11b (clone M1/70) (BD Biosciences, BDB561690); BV605-CD41 (clone MWReg30) (BioLegend, 133921); and PE/Cy7-CD71 (clone RI7217) (BioLegend, 113812). Viability was determined using DAPI (Invitrogen). Acquisition was achieved on an LSRII cytometer (BD Biosciences). Aggregates were excluded using the height and width of forward scatter and side scatter parameters. Analysis was performed using FlowJo software version 10.0.8 (Tree Star).

Frozen splenocytes were thawed and dead cells were depleted using the Dead Cell Removal Kit with LS columns (Miltenyi Biotec). Negative fractions were stained in 1\% BSA/2 mM EDTA buffer with Fixable Viability Dye eFluor 780 (eBioscience), PE-CD41 (BD Biosciences, clone HIP8), and APC-CD42 (Biolegend, clone 1C2) antibody conjugates and sorted on an Aria II Special Order flow cytometer (Beckton Dickinson). Data were analyzed and graphed using FlowJo software version 10.0.8.

Statistics. Statistical analyses were conducted using GraphPad Prism software v6.04 and the R software package (https://www.r-project.org). Differences between groups were compared using the unpaired 2-tailed Student's $t$ test with Welch's correction for unequal variance. One-way ANOVA, followed by Tukey's honest significant difference (HSD) multiple comparison test, was performed on datasets when comparing multiple groups. ANOVA, followed by Dunnett's multiple comparison test, was performed when comparing multiple groups with a single group in a dataset. All tests were evaluated at a statistical significance of 0.05 , if not otherwise specified.

Study approval. Animal experiments regarding $M P L^{W 515 L}$ mice were conducted in the H. Lee Moffitt Cancer Center and Research Institute in accordance with IACUC protocols (IS00001729) approved by University of South Florida IACUC. Animal experiments regarding JAK2 $2^{V 617 F}$ mice were conducted in Oklahoma University Health Sciences Center, and the experiments were performed in accordance with animal protocols (15-122-HW) approved by Oklahoma University IACUC.

\section{Author contributions}

L. Yue conducted the MSC studies and experiments with $M P L^{W 515 L}$ mice, analyzed data, and wrote the manuscript. Y. Han established the transplantation model. M. Bartenstein performed human sample analysis, megakaryocyte sorting, and TGF- $\beta$ production and wrote the corresponding part of the manuscript. W. Zhao and W. T. Ho conducted experiments with $J A K 2^{V 617 F}$ mice and wrote the corresponding part. C. Murdun performed virus production and drug treatment in $M P L^{W 515 L}$ mice. A. Mailloux assisted with MSC culture and IF staining. L. Zhang performed histological evaluation. X. Wang conducted statistical analyses. A. Budhathoki and K. Pradhan performed experiments and statistical analysis. F. Rapaport and RL Levine provided human sample data. U. Steidl, H. Wang, X. Ren and Z. Shao assisted with the studies. Z. Zhao, A. Verma, and P. Epling-Burnette together directed the project, analyzed data, provided conceptual input, and wrote the manuscript. 


\section{Acknowledgments}

We would like to thank Gary W. Reuther for critical assistance in retrovirus production. We also thank the Microscopy Core and Flow Cytometry Core at the H. Lee Moffitt Cancer Center for assistance with experiments. This work has been supported in part by the Biostatistics Core Facility at the H. Lee Moffitt Cancer Center and Research Institute (P30-CA076292). The studies were supported by the MPN Foundation and Leukemia Lymphoma Society. Amit Verma was supported by the Gottesman Stem Institute.

Address correspondence to: Pearlie K. Epling-Burnette, Department of Immunology, Malignant Hematology Division, SRB 23033, H. Lee Moffitt Cancer Center, Tampa, Florida 33612, USA. Phone: 813.745.6177; E-mail: Pearlie.Burnette@moffit.org. Or to: Amit Verma, Department of Medicine and Department of Developmental \& Molecular Biology, Albert Einstein College of Medicine, Jack and Pearl Resnick Campus, 1300 Morris Park Avenue, Chanin Building, Room 302B, New York, New York 10461, USA. Phone: 718.430.8761; E-mail: amit.verma@einstein.yu.edu. Or to: Zhizhuang Joe Zhao, University of Oklahoma Health Sciences Center, Department of Pathology, 940 Stanton L. Young Blvd, BMSB 451, Oklahoma City, Oklahoma 73104, USA. Phone: 405.271.9344; E-mail: Joe-Zhao@ouhsc.edu.

1. Tefferi A, Vardiman JW. Classification and diagnosis of myeloproliferative neoplasms: the 2008 World Health Organization criteria and point-of-care diagnostic algorithms. Leukemia. 2008;22(1):14-22.

2. Kralovics R, et al. A gain-of-function mutation of JAK2 in myeloproliferative disorders. N Engl J Med. 2005;352(17):1779-1790.

3. Tefferi A. Novel mutations and their functional and clinical relevance in myeloproliferative neoplasms: JAK2, MPL, TET2, ASXL1, CBL, IDH and IKZF1. Leukemia. 2010;24(6):1128-1138.

4. Klampfl T, et al. Somatic mutations of calreticulin in myeloproliferative neoplasms. N Engl J Med. 2013;369(25):2379-2390.

5. Tefferi A. Pathogenesis of myelofibrosis with myeloid metaplasia. J Clin Oncol. 2005;23(33):8520-8530.

6. Schmitt-Graeff AH, Nitschke R, Zeiser R. The hematopoietic niche in myeloproliferative neoplasms. Mediators Inflamm. 2015;2015:347270.

7. Tefferi A, et al. One thousand patients with primary myelofibrosis: the mayo clinic experience. Mayo Clin Proc. 2012;87(1):25-33

8. Cerquozzi S, Farhadfar N, Tefferi A. Treatment of myelofibrosis: a moving target. Cancer J. 2016;22(1):51-61

9. Lataillade JJ, et al. Does primary myelofibrosis involve a defective stem cell niche? From concept to evidence. Blood. 2008;112(8):3026-3035.

10. Walkley CR, Shea JM, Sims NA, Purton LE, Orkin SH. Rb regulates interactions between hematopoietic stem cells and their bone marrow microenvironment. Cell. 2007;129(6):1081-1095.

11. Schepers K, et al. Myeloproliferative neoplasia remodels the endosteal bone marrow niche into a self-reinforcing leukemic niche. Cell Stem Cell. 2013;13(3):285-299.

12. Kreipe H, Büsche G, Bock O, Hussein K. Myelofibrosis: molecular and cell biological aspects. Fibrogenesis Tissue Repair. 2012;5(Supp1 1):S21.

13. Varricchio L, Mancini A, Migliaccio AR. Pathological interactions between hematopoietic stem cells and their niche revealed by mouse models of primary myelofibrosis. Expert Rev Hematol. 2009;2(3):315-334.

14. Mailloux AW, et al. Fibrosis and subsequent cytopenias are associated with basic fibroblast growth factor-deficient pluripotent mesenchymal stromal cells in large granular lymphocyte leukemia. J Immunol. 2013;191(7):3578-3593.

15. Ponce CC, de Lourdes Lopes Ferrari Chauffaille M, Ihara SS, Silva MR. Increased angiogenesis in primary myelofibrosis: latent transforming growth factor- $\beta$ as a possible angiogenic factor. Rev Bras Hematol Hemoter. 2014;36(5):322-328.

16. Chagraoui H, Komura E, Tulliez M, Giraudier S, Vainchenker W, Wendling F. Prominent role of TGF-beta 1 in thrombopoietin-induced myelofibrosis in mice. Blood. 2002;100(10):3495-3503.

17. Zingariello $\mathrm{M}$, et al. Characterization of the TGF- $\beta 1$ signaling abnormalities in the Gata1low mouse model of myelofibrosis. Blood. 2013;121(17):3345-3363.

18. Ceglia I, et al. Preclinical rationale for TGF- $\beta$ inhibition as a therapeutic target for the treatment of myelofibrosis. Exp Hematol. 2016;44(12):1138-1155.e4

19. Leask A, Abraham DJ. TGF-beta signaling and the fibrotic response. FASEB J. 2004;18(7):816-827.

20. Pohlers D, et al. TGF-beta and fibrosis in different organs - molecular pathway imprints. Biochim Biophys Acta. 2009;1792(8):746-756.

21. Meindl-Beinker NM, Matsuzaki K, Dooley S. TGF- $\beta$ signaling in onset and progression of hepatocellular carcinoma. Dig Dis. 2012;30(5):514-523

22. Vannucchi AM, et al. A pathobiologic pathway linking thrombopoietin, GATA-1, and TGF-beta1 in the development of myelofibrosis. Blood. 2005;105(9):3493-3501.

23. Le Bousse-Kerdilès MC, Martyré MC, French INSERM research network on Idiopathic Myelofibrosis. Involvement of the fibrogenic cytokines, TGF-beta and bFGF, in the pathogenesis of idiopathic myelofibrosis. Pathol Biol. 2001;49(2):153-157.

24. Ciurea SO, et al. Pivotal contributions of megakaryocytes to the biology of idiopathic myelofibrosis. Blood. 2007;110(3):986-993.

25. Schmitt A, Jouault H, Guichard J, Wendling F, Drouin A, Cramer EM. Pathologic interaction between megakaryocytes and polymorphonuclear leukocytes in myelofibrosis. Blood. 2000;96(4):1342-1347.

26. Herbertz S, et al. Clinical development of galunisertib (LY2157299 monohydrate), a small molecule inhibitor of transforming growth factor-beta signaling pathway. Drug Des Devel Ther. 2015;9:4479-4499. 
27. Rampal R, et al. Integrated genomic analysis illustrates the central role of JAK-STAT pathway activation in myeloproliferative neoplasm pathogenesis. Blood. 2014;123(22):e123-e133.

28. Rodon J, et al. First-in-human dose study of the novel transforming growth factor- $\beta$ receptor I kinase inhibitor LY2157299 monohydrate in patients with advanced cancer and glioma. Clin Cancer Res. 2015;21(3):553-560.

29. Bhola NE, et al. TGF- $\beta$ inhibition enhances chemotherapy action against triple-negative breast cancer. J Clin Invest. 2013;123(3):1348-1358.

30. Zhou L, et al. Reduced SMAD7 leads to overactivation of TGF-beta signaling in MDS that can be reversed by a specific inhibitor of TGF-beta receptor I kinase. Cancer Res. 2011;71(3):955-963.

31. Li C, et al. Noncanonical STAT3 activation regulates excess TGF- $\beta 1$ and collagen I expression in muscle of stricturing Crohn's disease. J Immunol. 2015;194(7):3422-3431.

32. Laklai H, et al. Genotype tunes pancreatic ductal adenocarcinoma tissue tension to induce matricellular fibrosis and tumor progression. Nat Med. 2016;22(5):497-505.

33. Prêle CM, Yao E, O’Donoghue RJ, Mutsaers SE, Knight DA. STAT3: a central mediator of pulmonary fibrosis? Proc Am Thorac Soc. 2012;9(3):177-182.

34. Thiele J, Kvasnicka HM, Facchetti F, Franco V, van der Walt J, Orazi A. European consensus on grading bone marrow fibrosis and assessment of cellularity. Haematologica. 2005;90(8):1128-1132.

35. Zhao W, Ho WT, Zhao ZJ. Quantitative analyses of myelofibrosis by determining hydroxyproline. Stem Cell Investig. $2015 ; 2: 2$.

36. Xing S, et al. Transgenic expression of JAK2V617F causes myeloproliferative disorders in mice. Blood. 2008;111(10):5109-5117.

37. Wen QJ, et al. Targeting megakaryocytic-induced fibrosis in myeloproliferative neoplasms by AURKA inhibition. Nat Med. 2015;21(12):1473-1480.

38. Agarwal A, Morrone K, Bartenstein M, Zhao ZJ, Verma A, Goel S. Bone marrow fibrosis in primary myelofibrosis: pathogenic mechanisms and the role of TGF- $\beta$. Stem Cell Investig. 2016;3:5

39. Spangrude GJ, et al. P-selectin sustains extramedullary hematopoiesis in the Gata1 low model of myelofibrosis. Stem Cells. 2016;34(1):67-82.

40. Zingariello $\mathrm{M}$, et al. A novel interaction between megakaryocytes and activated fibrocytes increases TGF- $\beta$ bioavailability in the Gata1 (low) mouse model of myelofibrosis. Am J Blood Res. 2015;5(2):34-61.

41. Hong SH, et al. Rescue of a primary myelofibrosis model by retinoid-antagonist therapy. Proc Natl Acad Sci USA 2013;110(47):18820-18825.

42. Ponce CC, de Lourdes F Chauffaille M, Ihara SS, Silva MR. The relationship of the active and latent forms of TGF- $\beta 1$ with marrow fibrosis in essential thrombocythemia and primary myelofibrosis. Med Oncol. 2012;29(4):2337-2344.

43. Shehata M, Schwarzmeier JD, Hilgarth M, Hubmann R, Duechler M, Gisslinger H. TGF-beta1 induces bone marrow reticulin fibrosis in hairy cell leukemia. J Clin Invest. 2004;113(5):676-685.

44. Frenette PS, Pinho S, Lucas D, Scheiermann C. Mesenchymal stem cell: keystone of the hematopoietic stem cell niche and a stepping-stone for regenerative medicine. Annu Rev Immunol. 2013;31:285-316.

45. Uccelli A, Moretta L, Pistoia V. Mesenchymal stem cells in health and disease. Nat Rev Immunol. 2008;8(9):726-736.

46. Keating A. Mesenchymal stromal cells: new directions. Cell Stem Cell. 2012;10(6):709-716.

47. Méndez-Ferrer S, et al. Mesenchymal and haematopoietic stem cells form a unique bone marrow niche. Nature. 2010;466(7308):829-834.

48. García-García A, de Castillejo CL, Méndez-Ferrer S. BMSCs and hematopoiesis. Immunol Lett. 2015;168 (2):129-135.

49. Shen Y, Nilsson SK. Bone, microenvironment and hematopoiesis. Curr Opin Hematol. 2012;19(4):250-255.

50. Schneider RK, et al. Activated fibronectin-secretory phenotype of mesenchymal stromal cells in pre-fibrotic myeloproliferative neoplasms. J Hematol Oncol. 2014;7:92.

51. Avanzini MA, et al. Functional and genetic aberrations of in vitro-cultured marrow-derived mesenchymal stromal cells of patients with classical Philadelphia-negative myeloproliferative neoplasms. Leukemia. 2014;28(8):1742-1745.

52. Martinaud C, et al. Osteogenic potential of mesenchymal stromal cells contributes to primary myelofibrosis. Cancer Res. 2015;75(22):4753-4765.

53. Han Y, et al. Mesenchymal cell reprogramming in experimental MPLW515L mouse model of myelofibrosis. PLoS One. 2017;12(1):e0166014.

54. Verstovsek S, et al. Role of neoplastic monocyte-derived fibrocytes in primary myelofibrosis. J Exp Med. 2016;213(9):1723-1740

55. Biernacka A, Dobaczewski M, Frangogiannis NG. TGF- $\beta$ signaling in fibrosis. Growth Factors. 2011;29(5):196-202.

56. Flanders KC. Smad3 as a mediator of the fibrotic response. Int J Exp Pathol. 2004;85(2):47-64.

57. Schnabl B, Kweon YO, Frederick JP, Wang XF, Rippe RA, Brenner DA. The role of Smad3 in mediating mouse hepatic stellate cell activation. Hepatology. 2001;34(1):89-100.

58. Zhao J, et al. Smad3 deficiency attenuates bleomycin-induced pulmonary fibrosis in mice. Am J Physiol Lung Cell Mol Physiol. 2002;282(3):L585-L593.

59. Flanders KC, et al. Mice lacking Smad3 are protected against cutaneous injury induced by ionizing radiation. Am J Pathol. 2002;160(3):1057-1068.

60. Dobaczewski M, et al. Smad3 signaling critically regulates fibroblast phenotype and function in healing myocardial infarction Circ Res. 2010;107(3):418-428.

61. Bujak M, et al. Essential role of Smad3 in infarct healing and in the pathogenesis of cardiac remodeling. Circulation. 2007;116(19):2127-2138.

62. Saitoh M, et al. STAT3 integrates cooperative Ras and TGF- $\beta$ signals that induce Snail expression. Oncogene. 2016;35(8):1049-1057.

63. Liu RY, et al. JAK/STAT3 signaling is required for TGF- $\beta$-induced epithelial-mesenchymal transition in lung cancer cells. Int $J$ Oncol. 2014;44(5):1643-1651.

64. Liu M, et al. Immunomodulation by mesenchymal stem cells in treating human autoimmune disease-associated lung fibrosis. Stem Cell Res Ther. 2016;7(1):63.

65. Gastinne T, et al. Adenoviral-mediated TGF-beta1 inhibition in a mouse model of myelofibrosis inhibit bone marrow fibrosis development. Exp Hematol. 2007;35(1):64-74. 
66. Mascarenhas J, et al. Anti-transforming growth factor- $\beta$ therapy in patients with myelofibrosis. Leuk Lymphoma. 2014;55(2):450-452. 67. Manshouri T, et al. Bone marrow stroma-secreted cytokines protect JAK2 (V617F)-mutated cells from the effects of a JAK2 inhibitor. Cancer Res. 2011;71(11):3831-3840.

68. Pikman Y, et al. MPLW515L is a novel somatic activating mutation in myelofibrosis with myeloid metaplasia. PLoS Med. 2006;3(7):e270.

69. Lennon DP, Caplan AI. Isolation of human marrow-derived mesenchymal stem cells. Exp Hematol. 2006;34(11):1604-1605.

70. Santos AR, Duarte CB. Validation of internal control genes for expression studies: effects of the neurotrophin BDNF on hippocampal neurons. J Neurosci Res. 2008;86(16):3684-3692. 